\title{
One Size Does Not Fit All: Gender Inequity in STEM Varies Between Subfields
}

\author{
Stefanie Gisler, Anne E. Kato, Soohyun Lee, and Desmond W. Leung \\ Baruch College \& The Graduate Center, CUNY
}

We wholeheartedly agree with Miner et al. (2018) that industrial and organizational (I-O) psychologists should take a lead in addressing gender inequity in science, technology, engineering, and mathematics (STEM) fields. The focal article is particularly timely in light of the recent controversial "Google memo" (Damore, 2017), in which a senior software engineer endorsed the same individual-level myths regarding the gender gap in STEM that were critiqued by Miner et al. (2018). However, we caution against painting all STEM fields with the same broad brush. We argue that it is critical for I-O psychologists to be aware of important differences between STEM subfields, as these distinctions suggest that a "one-size-fits-all" approach may be inadequate for addressing existing gender disparities in STEM. In order to be maximally effective, interventions may need to emphasize distinct issues and target different points in the career pipeline depending on the specific STEM subfield in question.

\section{Gender Disparities Across STEM Subfields}

Disaggregating STEM and considering how gender inequity transpires in each subfield should be the starting point for addressing the gender gap in STEM. Although STEM comprises numerous fields, we will focus on six major subfields that have received the most attention in the literature on gender disparity (Cheryan, Ziegler, Montoya, \& Jiang, 2017): engineering, physics, computer science, biological science, chemistry, and mathematics/statistics. As observed by Cheryan et al. (2017), women are underrepresented to varying degrees in different STEM fields, and the patterns of underrepresentation vary across the career pipeline. In the United States, women obtain approximately half the bachelor's degrees in fields such as biological

Stefanie Gisler, Department of Psychology, Baruch College \& The Graduate Center, CUNY; Anne E. Kato, Department of Psychology, Baruch College \& The Graduate Center, CUNY; Soohyun Lee, Department of Psychology, Baruch College \& The Graduate Center, CUNY; Desmond W. Leung, Department of Psychology, Baruch College \& The Graduate Center, CUNY.

All authors contributed equally to this article. The order of authorship was determined alphabetically.

Correspondence concerning this article should be addressed to Stefanie Gisler, Department of Psychology, Baruch College \& The Graduate Center, CUNY, One Bernard Baruch Way, New York, NY 10010. E-mail: stefanie.gisler@baruch.cuny.edu 
sciences, chemistry, and mathematics/statistics but less than $20 \%$ of the bachelor's degrees in fields such as engineering, physics, and computer science (NSF, 2017). Most STEM fields have seen an increase in the proportion of women obtaining bachelor's degrees over the past few decades, but the rate of growth has varied across subfields. For example, between 1985 and 2013 , there was $11 \%$ growth in the proportion of women obtaining biological sciences degrees but only $5 \%$ growth in the proportion of women obtaining engineering degrees. In contrast to other fields, computer science has experienced a marked decline in the proportion of women obtaining bachelor's degrees since the 1980s.

Data from later stages of the career pipeline also reveal unique patterns of underrepresentation among STEM subfields. In the United States, women represent $47 \%$ of mathematical workers and $41 \%$ of life and physical scientists, but only $27 \%$ of computer workers and $13 \%$ of engineers (Landivar, 2013). Differential underrepresentation also exists between subfields within graduate degree programs. In fields in which women are fairly wellrepresented at the bachelor's degree level, such as biological sciences, chemistry, and mathematics/statistics, the proportion of women decreases at each subsequent level of education, with the lowest proportion completing doctoral degrees (NSF, 2017). This suggests that the major issue in these fields is retaining women through the talent pipeline. In contrast, in fields with significant underrepresentation of women at the bachelor's degree level, such as engineering, physics, and computer science, the proportion of women completing advanced degrees is greater than the proportion of women obtaining bachelor's degrees. This indicates that the major issue in these fields is one of recruitment, or attracting women to enter the field at an early career stage.

\section{Factors Contributing to Gender Disparities Within STEM Subfields}

The distinct patterns of gender differences across STEM subfields highlighted above suggest that the factors contributing to the existing gender inequity in STEM may be at least somewhat field specific. Thus, the development of successful interventions requires a more thorough understanding of the unique issues faced by each field. By examining the diversity of socialstructural issues that exist within and between STEM subfields, I-O psychologists may find that certain strategies aimed at achieving gender parity are better suited for some fields and less appropriate for others.

As the focal article highlighted, STEM is stereotyped as a highly masculine domain. The authors applied the lack of fit model (Heilman, 1983) to argue that women are widely viewed as unfit for STEM occupations due to their perceived lack of certain masculine attributes thought to be needed to succeed in these fields. However, the extent to which individual STEM fields are perceived as masculine varies. Generally speaking, heavily 
male-dominated fields such as computer science, engineering, and physics are perceived as more strongly associated with masculine stereotypes compared to relatively more gender-balanced fields such as biology and chemistry (Smyth \& Nosek, 2015). This would imply that women face greater challenges related to perceived lack of fit in certain STEM fields than in others.

Disaggregating STEM also reveals gender differences in formative educational experiences by subfield. For instance, although girls in high school and college are equally, if not more, likely to take courses in biology, chemistry, and math compared to their male peers, they are considerably less likely to enroll in physics, engineering, and computer science courses. There is also evidence of lower self-efficacy for women relative to men in STEM subjects that tend to be more male dominated (Cheryan et al., 2017). Given the variation in workforce gender ratios across STEM occupations, the availability of female role models also varies substantially between subfields. A lack of relatable role models in certain STEM subfields may prevent women and girls from envisioning the possibility of working in such fields. Thus, perceived lack of fit, schooling differences, lower self-efficacy, and lack of female role models may represent some of the biggest impediments for attracting women into highly male-dominated STEM fields. Conversely, retention issues in STEM subfields with relatively small gender disparities at the bachelor's degree level but wider gender disparities at subsequent levels may stem primarily from organization- or industry-level factors, such as unfair workplace practices, a lack of family-friendly policies, and/or workplace cultures that disproportionately impact women.

\section{Targeted Interventions Based on Subfields' Needs}

Although we endorse many of the practical recommendations outlined in the focal article for reducing gender disparities, we suggest that certain interventions may be better suited for some STEM subfields than for others. For example, internally focused organizational interventions such as job design and work-life balance initiatives may be most effective within relatively gender-balanced STEM fields. Focusing on increasing family-friendly policies (e.g., parental leave, flextime, telecommuting) may help these fields to address the key issue of retaining female talent beyond early career stages. Additionally, I-O psychologists should continue to advocate for fairer management practices (e.g., equity in pay and promotion) to increase women's opportunities for advancement in these fields.

Other approaches may be more beneficial in highly male-dominated STEM fields that face significant issues with attraction and recruitment. These fields may benefit most from interventions specifically geared toward recruiting women and girls during earlier stages of the pipeline. For example, educational partnerships and other outreach initiatives targeting middle 
school and high school students (e.g., summer camps, extracurricular programs) may help inspire girls' interest in STEM fields in which women are severely underrepresented. Additionally, given the lack of female role models in highly male-dominated STEM fields, one potential solution is to use the recruitment process to expose female students to a diverse range of role models with whom they can identify, including male role models who may be perceived as non-stereotypic (i.e., have fewer masculine traits). Deploying relatable role models to career fairs and other college recruiting events may contribute to increasing the representation of women in the recruiting pool and attenuating masculine stereotypes associated with male-dominated STEM fields.

In addition to issues related to attraction and recruitment, women working in (or considering working in) highly male-dominated STEM fields may be burdened with excessively high expectations for failure relative to their peers in other STEM subfields. Although we acknowledge that gender bias may be present in all STEM fields, it is likely to be especially prevalent within subfields that are more strongly associated with masculine stereotypes. Thus, in these fields, we argue that I-O psychologists should pay particularly close attention to gender bias that may exist in organizational practices, such as selection and performance appraisal systems

\section{Conclusion}

In order to take the lead in addressing the gender gap in STEM, I-O psychologists should move beyond a monolithic view of STEM and examine how various factors-both inside and outside the workplace-contribute to gender disparities within and between STEM subfields. We have highlighted some differences between core STEM disciplines; future work should continue and expand this line of inquiry to other subfields that have received less attention in the literature, such as health and social sciences. Disaggregating and broadening our conceptualization of STEM may help I-O psychologists critically evaluate current interventions and identify new approaches for reducing gender disparities in STEM.

\section{References}

Cheryan, S., Ziegler, S. A., Montoya, A. K., \& Jiang, L. (2017). Why are some STEM fields more gender balanced than others? Psychological Bulletin, 143(1), 1-35.

Damore, J. (2017, July). Google's ideological echo chamber. Retrieved from https://assets. documentcloud.org/documents/3914586/Googles-Ideological-Echo-Chamber.pdf

Heilman, M. E. (1983). Sex bias in work settings: The lack of fit model. Research in Organizational Behavior, 5, 269-298.

Landivar, L. C. (2013). Disparities in STEM employment by sex, race, and Hispanic origin. (American Community Survey Reports ACS-24). Washington, DC: U.S. Census Bureau. Retrieved from https://www.census.gov/prod/2013pubs/acs-24.pdf. 
Miner, K. N., Walker, J. M., Bergman, M. E., Jean, V. A., Cater-Sowell, A., January, S. C., \& Kaunas, C. (2018). From "her" problem to "our" problem: Using an individual lens versus a social-structural lens to understand gender inequity in STEM. Industrial and Organizational Psychology: Perspectives on Science and Practice, 11(2), 267-290.

National Science Foundation, National Center for Science and Engineering Statistics. (2017). Women, Minorities, and Persons with Disabilities in Science and Engineering: 2017. Special Report NSF 17-310. Retrieved from https://www.nsf.gov/statistics/2017/nsf17310/digest/about-this-report/ Smyth, F. L., \& Nosek, B. A. (2015). On the gender-science stereotypes held by scientists: Explicit accord with gender-ratios, implicit accord with scientific identity. Frontiers in Psychology, 6, 415.

\title{
Yes Virginia, There Is a Gender Disparity Problem-and It Goes Beyond STEM
}

\author{
Satoris S. Howes \\ Oregon State University \\ Jaime Henning \\ Eastern Kentucky University \\ Maura J. Mills \\ University of Alabama \\ Ann Hergatt Huffman \\ Northern Arizona University
}

Miner et al. (2018) do an excellent job of bringing the issue of gender disparity within STEM to the forefront of I-O psychology. However, we believe the focus on STEM is woefully inadequate and urge I-O psychologists to think bigger, better, and broader. There are clear problems with the way women are viewed and treated within the workforce, within the United States, and globally. In narrowing the discussion of the problem to target only STEM, we dramatically limit our understanding of and potential impact on the multifaceted and complex gender disparity problem in the world of work. Furthermore, we assert there are additional legitimizing myths that must be addressed in order to yield a more complete picture of the dilemma and allow us to move forward to make an impact.

We are not arguing that I-O psychologists should not care about the gender disparity in STEM. Of course we should. But, as workplace scholars, do we not have a responsibility toward addressing gender disparity in other

Satoris S. Howes, Oregon State University, Cascades Campus; Jaime Henning, Eastern Kentucky University; Maura J. Mills, Department of Management, University of Alabama; Ann Hergatt Huffman, Northern Arizona University.

Correspondence concerning this article should be addressed to Satoris S. Howes, Oregon State University-Cascades, 1500 SW Chandler Ave, Bend, OR 97702. E-mail: satoris.howes@gmail.com 